\title{
X-ray Diffraction (XRD) and Energy Dispersive Spectroscopy (EDS) Analysis of Silver Nanoparticles Synthesized from Erythrina Indica Flowers
}

\author{
Amargeetha $\mathbf{A}^{1}$ and Velavan $\mathbf{S}^{2^{*}}$ \\ ${ }^{1}$ Research Scholar, P.G and Research Department of Biochemistry, Marudupandiyar College, Thanjavur, Tamil Nadu, India \\ ${ }^{2}$ Associate Professor, P.G and Research Department of Biochemistry, Marudupandiyar College, Thanjavur, Tamil Nadu, India
}

Received: December 7, 2017; Accepted: December 20, 2017; Published: February 5, 2018

*Corresponding author: Velavan S, Associate Professor, P.G and Research Department of Biochemistry, Marudupandiyar College, Thanjavur, Tamil Nadu, India E-mail: mayavelvan@gmail.com

\begin{abstract}
Nanoparticles usually speak of particles with a size up to 100 $\mathrm{nm}$ with increasing in surface to volume ratio, providing more active surface atoms to contribute the role in applications and improve the properties of the materials1. The control particle size, particle shape and morphology are very much important in nanoparticles preparation. The most important tool to study the nano materials is X-ray diffraction. X-ray diffraction is one of the most important characterization tools used in solid state chemistry and materials science. XRD is an easy tool to determine the size and the shape of the unit cell for any compound. In this study explaining the formation of silver nanoparticles through Erythrina indica flowers. The synthesized AgNPs further examined the X-ray Diffraction (XRD) and Energy Dispersive Spectroscopy (EDS) analysis. The X-ray diffraction results clearly show that the silver nanoparticles formed by the reduction of Ag+ ions by the Erythrina indica flower extract are crystalline in nature. The average crystalline size of AgNPs is calculated using Debye-Scherrer formula is $\sim 28.19 \mathrm{~nm}$. The EDX reading proved that the required phase of silver $(\mathrm{Ag})$ is present in the sample.
\end{abstract}

Keywords: Erythrina Indica Flower; X-Ray Diffraction; Energy Dispersive Spectroscopy; Silver Nanoparticles

\section{Introduction}

Nanoparticles usually speak of particles with a size up to 100 $\mathrm{nm}$ with increasing in surface to volume ratio, providing more active surface atoms to contribute the role in applications and improve the properties of the materials [1]. The control particle size, particle shape and morphology are very much important in nanoparticles preparation. The most important tool to study the nano materials is X-ray diffraction. X-ray diffraction is one of the most important characterization tools used in solid state chemistry and materials science. XRD is an easy tool to determine the size and the shape of the unit cell for any compound. Silver nanoparticles with particle size are prepared in room temperature and the results are confirmed by XRD. A variety of methods have been adopted to make nanoparticles on solid surfaces, including diverse lithographic techniques, deposition of a metal colloid, controlled nanoparticles growth by diffusion, vacuum deposition of metal, electrophoretic chemical and electrochemical deposition of metal nanoparticles, etc. For uniform size, shape and spacing of nanoparticles, there are various techniques like Lithographic and vacuum deposition of metal, but expensive techniques [2-5]. One of the suitable, simplest and low-cost methods is co-precipitation method which can be used in wide range of materials. In this study explaining the formation of silver nanoparticles through Erythrina indica flowers. The synthesized AgNPs further examined the X-ray Diffraction (XRD) and Energy Dispersive Spectroscopy (EDS) analysis.

\section{Materials and Methods}

\section{Chemicals}

All the experiments were conducted at room temperature. Materials used for the synthesis of silver nanoparticles are AR grade silver nitrate $\left(\mathrm{AgNO}_{3}\right)$ purchased from Merck, India.

\section{Collection of Plant Materials}

The mature Erythrina indica flowers were collected in April 2015 from Kodaikanal, Dindugal district, Tamil Nadu, India. The flower were identified and authenticated by Botanist, Prof. Dr. S. John Britto, Director, The Rapinat Herbarium, St. Josephs College, Tiruchirappalli, Tamil Nadu, India.

\section{Synthesis of Ag Nanoparticles using Flower Extracts}

The dried flowers were pulverized well with mortar and pestle to make a powder. Twenty grams of powder sample was mixed into $100 \mathrm{ml}$ of deionized water and the mixture was boiled for $10 \mathrm{~min}$. After cooling the flower extract was filtered with Whatman No. 1 filter paper. The filtrate was stored at $4^{0} \mathrm{C}$ for further use.

For the Ag nanoparticles synthesis, $5 \mathrm{ml}$ of Erythrina indica flower extract was added to $45 \mathrm{ml}$ of $1 \mathrm{mM}$ aqueous $\mathrm{AgNO}_{3}$ solution 
in a $250 \mathrm{ml}$ Erlenmeyer flask. The flask was then incubated in the dark at $5 \mathrm{hrs}$ (to minimize the photo activation of silver nitrate), at room temperature. A control setup was also maintained without flower extract. The Ag nanoparticles solution thus obtained was purified by repeated centrifugation at $10,000 \mathrm{rpm}$ for $15 \mathrm{~min}$ followed by re-dispersion of the pellet in de-ionized water. Then the Ag nanoparticles were freeze dried using SEM analysis [4].

\section{Diffraction Method}

The phase evolution of calcined powder as well as that of sintered samples was studied by X-ray diffraction technique (Philips PAN analytical, The Netherland) using $\mathrm{Cu}$ Ká radiation. The generator voltage and current was set at $35 \mathrm{KV}$ and $25 \mathrm{~mA}$ respectively. The Ag samples were scanned in the 2è ranges 15 to $700{ }^{\circ} \mathrm{C}$ range in continuous scan mode. The scan rate was $0.04^{\circ} /$ sec.

\section{EDX Analysis}

EDX were used to find elemental composition in the reaction mixture. The EDX analysis of AgNPs sample was done by the SEM (JEOLJSM 5800) machine. The EDX normally reveals the presence of phases.

\section{Results}

\section{Synthesis of Silver Nanoparticles}

A study on phytosynthesis of Ag nanoparticles by the aqueous flower extract of Erythrina indica was carried out in this work. During the visual observation, silver nitrate incubated with flower extract showed a color change from yellow to brown within $5 \mathrm{~h}$ whereas no color change could be observed in silver nitrate without flower extract. The appearance of brown color in flower extract treated flask is clear indication for the formation of Ag nanoparticles). This color arises due to excitation of surface Plasmon vibrations in Ag nanoparticles. Previously reported that colour change was observed at 90 minutes from light green to light brown colour, indicating the formation of silver nanoparticles which also indicated the presence of silver nanoparticles. We have demonstrated the efficiency of Erythrina indica flower extract in the rapid synthesis of silver nanoparticles possessing a variety of fascinating morphologies owing to its diverse groups of phytochemicals like phenolics, favonoids, polyphenols, reducing sugars, anthraquinones, terpenoids and anthrones.

\section{XRD pattern of Ag NPs synthesized from Erythrina indica flowers}

XRD is commonly used for determining the chemical composition and crystal structure of a material; therefore, detecting the presence of silver nanoparticles in plants tissues can be achieved by using XRD to examine the diffraction peaks of the AgNPs synthesized from Erythrina indica flower extract.

X-ray diffraction pattern of the biosynthesized Ag NPs from the leaf extract is shown in Fig. 1.The crystalline nature of $\mathrm{Ag}$ nanoparticles was further confirmed from X-ray diffraction (XRD) analysis shows the XRD pattern of the dried nanoparticles obtained from colloid samples. A number of Bragg reflections with $2 \varnothing$ values of $24.934,37.0359$ and $43.8572^{\circ}$ indicate the (100), (200) and (300) reflections of metallic silver clearly indicate the cubic crystalline face-centered cubic structure of silver and compared with the standard powder diffraction card of JCPDS, silver file No. 04-0783. Here, the constant is 54.27 (100.87 - $46.60=54.27$ ). The unassigned peaks could be due to the crystallization of bioorganic phase that occurs on the surface of the nanoparticles. The observed peak broadening and noise were probably macromolecules present in the plant extract which may be responsible for the reduction of silver ions. Hence XRD pattern thus clearly illustrated that the silver nanoparticles formed in this present synthesis are crystalline in nature. In addition to the Bragg peaks representative of silver nanocrystals, additional as yet unassigned peaks are also observed suggesting that the crystallization of bio-organic phase occurs on the surface of the silver nanoparticles. The line broadening of the peaks is primarily due to small particle size. Indexing has been done and data are in Table 1.

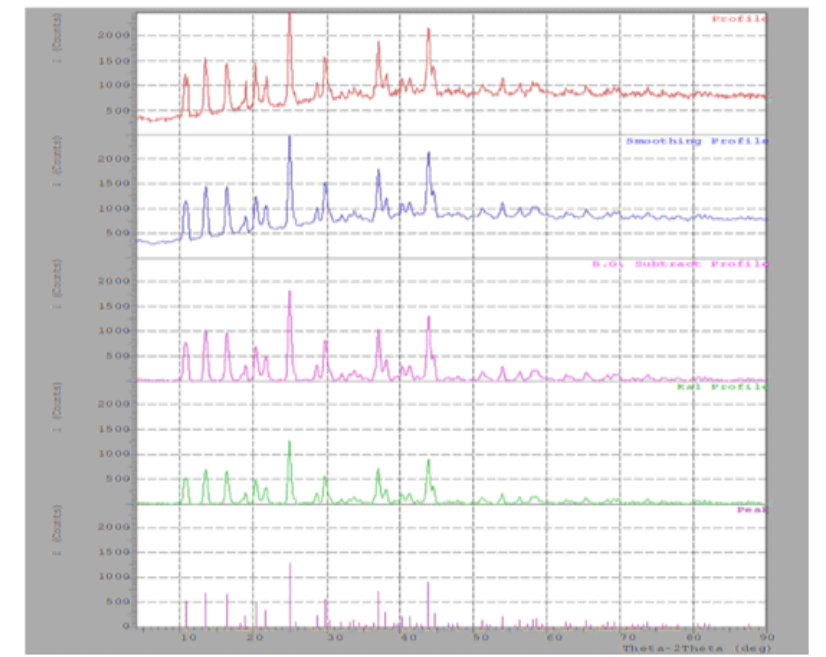

Figure 1: SXRD pattern of silver nanoparticles synthesized by Erythrina indica flowers

Table 1: XRD pattern of silver nanoparticles synthesized by Erythrina indica flowers Simple peak indexing

\begin{tabular}{|c|c|c|c|c|}
\hline Peak position 20 & $\mathbf{1 0 0 0} \times \mathbf{S i n} \mathbf{2 \theta}$ & $\mathbf{1 0 0 0} \times \mathbf{S i n} \mathbf{2 \theta} / \mathbf{5 4 . 2 7}$ & Reflection & Remarks \\
\hline 24.934 & 46.60321 & 1 & 100 & $1^{2}+0^{2}+0^{2}=1$ \\
\hline 37.0359 & 100.8713 & 2 & 200 & $1^{2}+1^{2}+0^{2}=2$ \\
\hline 43.8572 & 139.4662 & 3 & 300 & $1^{2}+1^{2}+1^{2}=3$ \\
\hline
\end{tabular}




\section{Particle Size Calculation}

From this study, considering the peak at degrees, average particle size has been estimated by using Debye-Scherrer formula [6-9]

$$
D=0.9 \lambda / \beta \operatorname{Cos} \theta
$$

Where ' $\lambda$ ' is wave length of X-Ray $(0.1541 \mathrm{~nm})$, ' $\beta$ ' is FWHM (full width at half maximum), ' $\theta$ ' is the diffraction angle and ' $D$ ' is particle diameter size. The average crystalline size according to to Debye-Scherrer equation calculated is found to be $28.19 \mathrm{~nm}$ and represent in Table 2.

\section{Energy Dispersive Spectroscopy (EDS) Analysis of AgNPs}

EDX were used to find elemental composition in the reaction mixture. EDS of AgNPs revealed the presence of pure silver (Ag 55.08\%) was the major constituent element compared to chloride $(44.92 \%)$ as Table 3 . The EDX reading proved that the required phase of silver (Ag) was present in the AgNPs (Figure 2).

Table 2: The grain size of silver nanoparticles

\begin{tabular}{|c|c|c|c|c|}
\hline $2 \theta$ of the intense peak (deg) & Miller indices (hkl) & $\theta$ of the intense peak (deg) & $\begin{array}{l}\text { FWHM of intense } \\
\text { peak }(\beta) \text { radians }\end{array}$ & $\begin{array}{l}\text { Size of the particle } \\
\text { (D) } \mathrm{nm}\end{array}$ \\
\hline 24.934 & 100 & 12.46 & 0.003754293 & 37.69 \\
\hline 37.0359 & 200 & 18.51 & 0.005640917 & 26.07 \\
\hline 43.8572 & 300 & 21.92 & 0.006679391 & 20.81 \\
\hline \multicolumn{4}{|c|}{ Average size of particles } & 28.19 \\
\hline
\end{tabular}

Table 3: \% of elements present in the AgNPs

\begin{tabular}{|c|c|c|c|c|}
\hline Elements & AN & Series & Weight \% & 78.86 \\
\hline $\mathrm{Ag}$ & 47 & L-series & 21.14 \\
\hline $\mathrm{Cl}$ & 17 & K-series & 100 \\
\hline
\end{tabular}

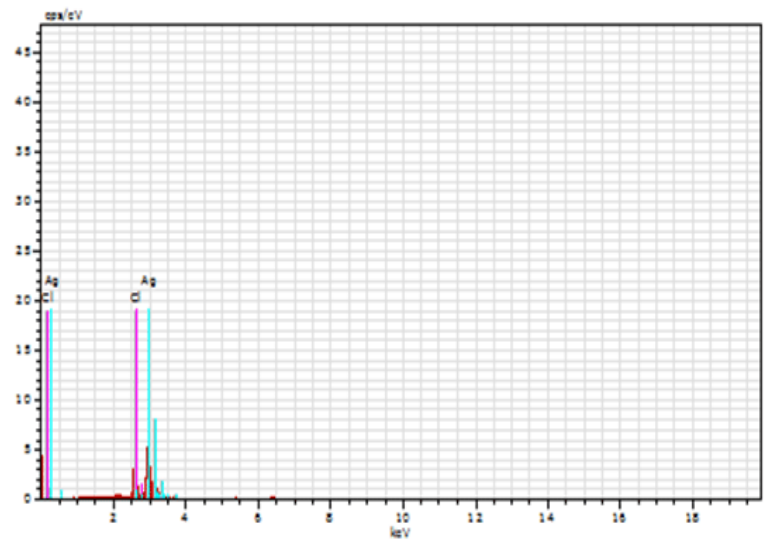

Figure 2: EDS-Spectroscopy view of the Erythrina indica showing synthesis of silver nanoparticles and elemental silver signal in higher percentage

\section{Discussion}

\section{X-ray diffraction (XRD) Analysis of AgNPs}

$\mathrm{XRD}$ is a useful tool in obtaining information about the atomic structure of materials. XRD is not only usually used for qualitative identification of minerals in geological samples by fingerprinting approach but also is used for the quantification of mineralogical data [10]. XRD is a valuable characterization tool to prove the formation of AgNPs, determine the crystal structure and calculate the crystalline nanoparticles size $[11,12]$.

X-ray diffraction pattern of the biosynthesized AgNPs from the flower extract was examined. The crystalline nature of Ag nanoparticles was confirmed from X-ray diffraction (XRD) analysis shows the XRD pattern of the dried nanoparticles obtained from colloid samples. Three peaks were observed at $24.934^{\circ}, 37.0359^{\circ}$ and $43.8572^{\circ}$ in the $2 \theta$ range $10-60^{\circ}$. These Braggs reflections clearly indicated that biosynthesized Ag NPs showed characteristic peaks indexed to the crystalline planes (100), (200) and (300) of (3.46), (2.16) and (2.32) sets of lattice planes and further on the basis that they can be indexed as FaceCentered-Cubic (FCC) structure of silver. The sharpness of the peak clearly indicates that the particles are in the nanoregime.

The observed peak broadening and noise were probably macromolecules present in the plant extract which may be responsible for the reduction of silver ions. Hence XRD pattern thus clearly illustrated that the silver nanoparticles formed in this present synthesis are crystalline in nature. In addition to the Bragg peaks representative of silver nanocrystals, additional as yet unassigned peaks are also observed suggesting that the crystallization of bio-organic phase occurs on the surface of the silver nanoparticles. The line broadening of the peaks is primarily due to small particle size. 
The X-ray diffraction results clearly show that the silver nanoparticles formed by the reduction of $\mathrm{Ag}+$ ions by the Erythrina indica flower extract are crystalline in nature. The average crystalline size of AgNPs is calculated using DebyeScherrer formula is $\sim 28.19 \mathrm{~nm}$. This results agreement with the earlier reports $[13,14]$. Neveen Abdel-Raouf demonstrated the pattern of AgNPs synthesized by Galaxaura elongate is coincides with results of plant mediated synthesis of AgNPs [15]. The broadening of Bragg's peaks around their bases indicates the formation of small sized silver nanoparticles [16-18]. A few unassigned peaks observed could be due to the presence of some bioorganic compounds/protein(s) in the leaf extract and crystallizes on the surface of the silver. Similar results silver nanoparticles synthesized using Mangifera indica extract, Geranium, mushroom extract, and Coleus aromaticus extract were reported [18, 19, 20, 21].

\section{Energy Dispersive Spectroscopy (EDS) Analysis of AgNPs}

EDX spectrum reveals strong signal in the silver region and confirms the formation of AgNPs. Metallic silver nanocrystals generally show typical optical absorption peak approximately at $3 \mathrm{KeV}$ due to surface plasmon resonance [22]. Silver ((Ag 55.08\%) was the major constituent element compared to chloride (44.92\%). The energy dispersive spectroscopy (EDX) data show very strong silver and weak signals of chloride and carbon peaks, which indicate that the reduction of silver ions to elemental silver possibly originated from the molecules attached to the surface of the AgNPs. The dense peak of silver strongly confirmed the reduction of silver nitrate to silver nanoparticles. Silver peak is thicker than other peak. This confirms the complete reduction of silver compounds to AgNPs as shown in the spectrum. The EDX reading proved that the required phase of silver $(\mathrm{Ag})$ is present in the sample.

\section{Conclusion}

The green synthesis method is eco-friendly low cost and capable of producing AgNPs at room temperature. Here, Erythrina indica flower extract act as both reducing and stabilizing agents. The AgNPs were confirmed the surface plasmon resonance of green-synthesized silver nanoparticles. The X-ray diffraction results clearly show that the silver nanoparticles formed by the reduction of $\mathrm{Ag}^{+}$ions by the Erythrina indica flower extract are crystalline in nature. The average crystalline size of AgNPs is calculated using Debye-Scherrer formula is $\sim 28.19 \mathrm{~nm}$. The EDX reading proved that the required phase of silver $(\mathrm{Ag})$ is present in the sample. Silver was the major constituent element compared to carbon and chloride. In this present study proved that the crystalline nature of silver present in the AgNPs confirmed by X-ray diffraction and EDX.

\section{References}

1. Willems, van den Wildenberg. Roadmap Report on Nanoparticles. W\&WE spanasl. Barcelona. Spain. 2005.

2. Bo Xu, Kevan Larry. Formation of Silver Ionic Clusters and Silver Metal Particles in Zeolite Rho Studied by Electron Spin Resonance and Far-Infrared Spectroscopies. Journal of Physical Chemistry B. 1991;95(3):1147-1151. doi:10.1021/j100156a023

3. Colby A Foss, Michael J Tierney, Charles R Martin. Template Synthesis of Infrared-Transparent Metal Microcylinders: Comparison of Optical Properties with the Predictions of Effective Medium Theory. Journal of Physical Chemistry B. 1992;96(22):9001-9007. doi:10.1021/ j100201a057

4. Colby A Foss, Gabor L Hornyak, Jon A Stockert, Charles R Martin. Template-Synthesized Nanoscopic Gold Particles: Optical Spectra and the Effects of Particle Size and Shape. Journal of Physical Chemistry B. 1994;98(11):2963-2971. doi:10.1021/j100062a037

5. Bo Xu, Larry Kevan. Formation of Alkali Metal Partides in Alkali Metal Cation Exchanged X Zeollte Exposed to Atka Metat Vapor: Control of Metat Particle Identity. Journal of Physical Chemistry B. 1992;96(6):2642-2645. doi:10.1021/j100185a046

6. Yugang Sun, Younan Xia. Shape-Controlled Synthesis of Gold and Silver Nanoparticles. Science. 2002;298(5601):2176-2179. doi:10.1126/ science.1077229

7. Nath S S , Chakdar D, Gope G. Synthesis of CdS and ZnS Quantum Dots and Their Applications in Electronics. Nanotrends-A . Journal of Nanotechnology and Its Application. 2007;2(3).

8. Nath S S, Chakdar D, Gope G, Avasthi D K. Effect of 100 Mev Nickel Inos on Silica Coated ZnS Quantum Dot. Journal of Nanoelectronics and Optoelectronics. 2008;3(2):180-184.

9. Stephen Brunauer, Emmett P H, Edward Teller. Adsorption of Gases in Multimolecular Layers. Journal of the American Chemical Society. 1938;60(2):309-319. doi:10.1021/ja01269a023

10.Said S Al-Jaroudi, AnwarUl-Hamid, Abdul-Rashid I Mohammed, Salih Saner. Use of X-ray powder diffraction for quantitative analysis of carbonate rock reservoir samples. Powder Technology. 2007;175(3):115-121.

11. Daizy Philip. Mangifera indica leaf-assisted biosynthesis of well dispersed silver nanoparticles. Spectrochimica Acta Part A: Molecular and Biomolecular Spectroscopy. 2011;78(1):327-331.

12. Sumitra Chanda. Silver nanoparticles (medicinal plants mediated): A new generation of antimicrobials to combat microbial pathogens- a review. In Méndez-Vilas, A. (Ed). Microbial pathogens and strategies for combating them: science, technology and education. Spain: Formatex. 2013;1314-1323.

13. Yuesheng Huang, Xiaolu Li, Zhenjiang Liao, Guoan Zhang, Qun Liu, Jin Tang, Yizhi Peng, Xuesheng Liu, Qizhi Luo. A randomized comparative trial between Acticoat and SD-Ag in the treatment of residual burn wounds, including safety analysis. Burns. 2007;33(2):161-166.

14. Udayasoorian C, Vinoth kumar K, Jayabalakrishnan R M. Extracellular synthesis of silver nanoparticles using leaf extract of cassia auriculata. Digest Journal of Nanomaterials and Biostructures. 2011;6(1):279283.

15. Vaveen Abdel-Raouf N, Al-Enazi N M, Ibraheem I B M. Green biosynthesis of gold nanoparticles using Galaxaura elongata and characterization of their antibacterial activity. Arabian Journal of Chemistry. 2013;11:44-49. 
16. Smitha S L, Philip D, Gopchandran K G. Green synthesis of gold nanoparticles using Cinnamomum zeylan- icum leaf broth. Spectrochimica Acta - Part A: Molecular and Biomolecular Spectroscopy. 2009;74(3):735-739.

17. GnanaJobitha G, Rajeshkumar S, Annadurai G, Kannan C. Preparation and characterization of fruit-mediated silver nanoparticles using pomegranate extract and assessment of its antimicrobial activities. Journal of Environmental Nanotechnology. 2013;2(1):4-10.

18. Mahendran Vanaja, Gurusamy Annadurai. Coleus aromaticus leaf extract mediated synthesis of silver nanoparticles and its bactericidal activity. Applied Nanoscience. 2013;3(3):217-223.
19. Daizy Philip. Mangifera indica leaf assisted biosynthesis of well dispersed silver nanoparticles. Spectrochimica Acta Part A: Molecular and Biomolecular Spectroscopy. 2011;78(1):327-331.

20. Shankar S S, Ahmad A, Sastry M. Geranium Leaf Assisted Biosynthesis of Silver Nanoparticles. Biotechnology Progress. 2003;19(6):16271631. doi:10.1021/bp034070w

21. Daizy Philip. Biosynthesis of $\mathrm{Au}, \mathrm{Ag}$ amd $\mathrm{Au}-\mathrm{Ag}$ nanoparticles using edible mushroom extract. Spectrochimica Acta Part A: Molecular and Biomolecular Spectroscopy. 2009;73(2):374-381.

22. Kaviya S, Santhanalakshmi J, Viswanathan B, Muthumar J, Srinivasan K. Biosynthesis of silver nanoparticles using Citrus sinensis peel extract and its antibacterial activity. Spectrochimica Acta Part A: Molecular and Biomolecular Spectroscopy. 2011;79(3):594-598. 